\title{
Occult nonmetallic intraocular foreign bodies presenting as fulminant uveitis: a case series and review of the literature
}

This article was published in the following Dove Press journal:

Clinical Ophthalmology

30 August 2013

Number of times this article has been viewed

\author{
Yevgeniy V Sychev' \\ Elizabeth A Verner-Cole ${ }^{2}$ \\ Eric B Suhler ${ }^{2}$ \\ J Timothy Stout ${ }^{2}$ \\ G Atmaram Vemulakonda' \\ 'Department of Ophthalmology, \\ University of Washington, Seattle, WA, \\ USA; ${ }^{2}$ Casey Eye Institute, Oregon \\ Health and Science University, \\ Portland, OR, USA
}

\begin{abstract}
Intraocular foreign bodies (IOFBs) can complicate globe trauma and are associated with a high incidence of severe vision loss. Occult IOFBs present a particular challenge as they are not diagnosed promptly and tend to present with advanced complications, including endophthalmitis and retinal detachment. In this report, we present three cases of occult nonmetallic IOFBs presenting as fulminant uveitis, and we also review the literature.
\end{abstract}

Keywords: intraocular foreign body, endophthalmitis, vitrectomy, trauma

\section{Introduction}

Intraocular foreign bodies (IOFBs) can complicate penetrating globe trauma and are associated with a high incidence of severe vision loss. ${ }^{1}$ Occult IOFBs present a particular challenge as by definition they are not diagnosed promptly and tend to present with advanced complications, including endophthalmitis and retinal detachment. ${ }^{2}$ In this report, we present three cases of occult nonmetallic IOFBs presenting as fulminant uveitis and review the literature. , $^{3,4}$

\section{Case I}

A 36 year old man presented with decreased vision, pain, epiphora, and photophobia in the left eye (OS) after being hit in the eye by a blackberry bush. An external ophthalmologist treated him with oral and topical corticosteroids and cyclopentolate before referring him 2 weeks later. His left eye had hand motions visual acuity (VA) and an intraocular pressure (IOP) of 14. Slit lamp examination revealed a small subconjunctival elevation inferotemporal to the limbus, 4+ pigmented anterior chamber (AC) cell, a fibrin clot, and dense vitritis precluding clear fundus visualization. B-scan ultrasonography identified a hyper-reflective area that is concerning for a foreign body (Figure 1). Examination of the right eye (OD) was unremarkable. It was decided to proceed with surgical intervention to address the presumed endophthalmitis and possible IOFB. The patient underwent vitrectomy, endolaser, and scleral buckle for a limited retinal detachment discovered intraoperatively in the area of the external injury. A small thorn which extended from the sclera, through the retina, and into the vitreous was discovered in this same area of the retinal detachment and was removed (Figures 2 and 3). He received intraoperative intravitreal injections of vancomycin, voriconazole, and ceftazidime. He was also treated postoperatively with topical atropine, polymyxin/ trimethoprim, and prednisolone acetate along with oral moxifloxacin $400 \mathrm{mg}$ daily and voriconazole $200 \mathrm{mg}$ twice daily. Vitreous polymerase chain reaction (PCR) and
Correspondence: Gurunadh Atmaram Vemulakonda

325 Ninth Ave, Box 359608, Seattle,

WA 98I04, USA

Tel +I 5033827997

Fax + I 2068974320

Email gatmav@gmail.com 


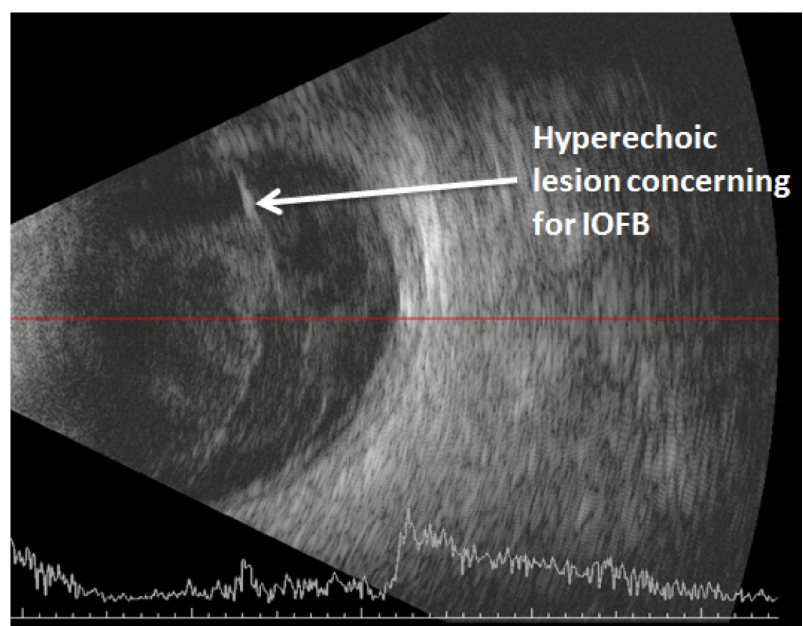

Figure I B-scan ultrasonography image of intravitreal thorn.

Notes: Areas of choroidal thickening, with diffuse, dense vitreous debris, along with a hyper-reflective lesion at the 4 o'clock hour.

Abbreviation: IOFB, intraocular foreign body.

cultures both revealed Staphylococcus epidermidis. Seven months following the initial surgery and 4 months following cataract removal and lens placement, the patient was pain-free with best corrected VA of 20/25+1

\section{Case 2}

A 10 year old boy presented with 2 weeks of pain, pressure sensation, photophobia, and redness without discharge in the left eye and a VA of 20/150. He was initially treated with topical and systemic corticosteroids. Despite treatment, his symptoms progressed and after one week his VA had worsened. He was then referred to a tertiary care center. Despite extensive questioning of both parents and patient, the patient denied any history of trauma, and the patient had no systemic symptoms. Examination of his right eye was unremarkable. His left eye had a VA of 20/300 and an IOP of 5.

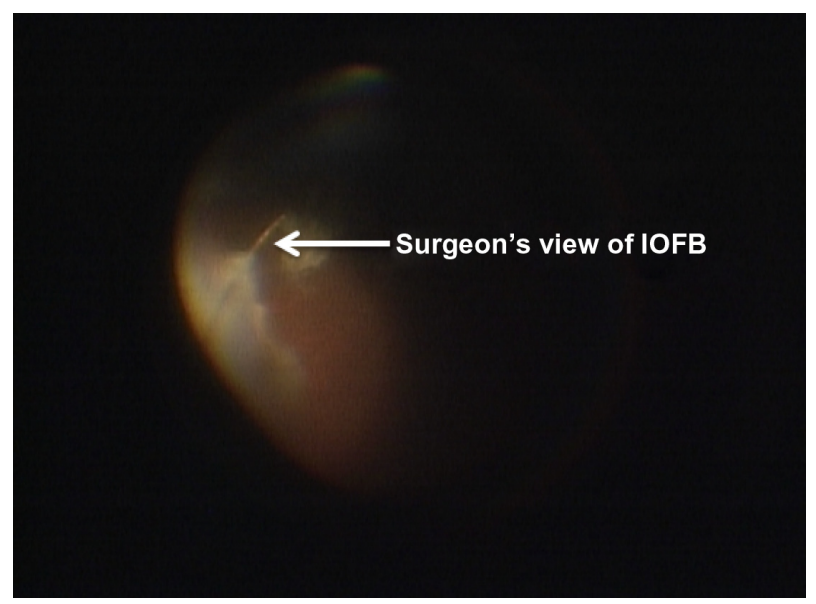

Figure 2 Intraoperative image of intravitreal thorn. Abbreviation: IOFB, intraocular foreign body.

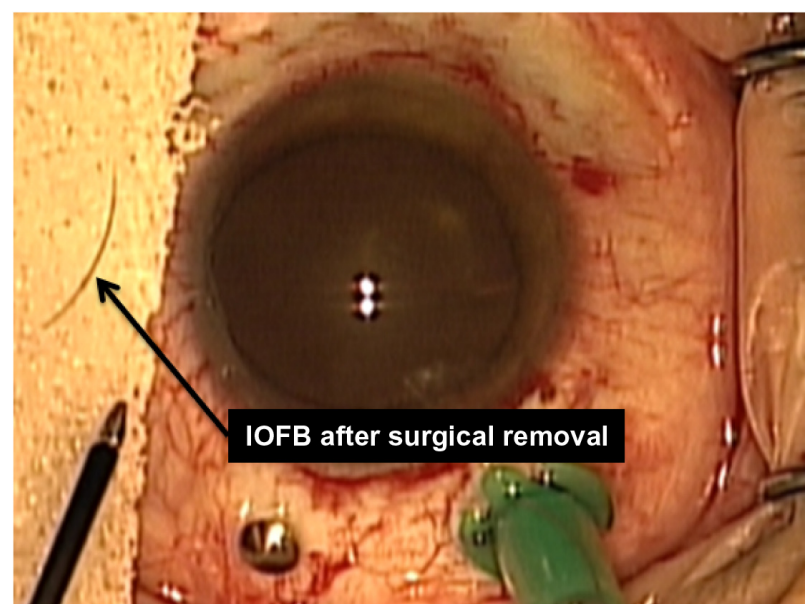

Figure 3 Photographic still of thorn following removal. Abbreviation: IOFB, intraocular foreign body.

Examination of his left eye revealed conjunctival injection, 4+ $\mathrm{AC}$ cell, and $0.1 \mathrm{~mm}$ hypopyon. Fundus detail was obscured by vitreous haze. B-scan ultrasound was remarkable for mild vitritis but did not demonstrate a foreign body. The patient underwent an investigation for infectious and inflammatory causes including Herpes simplex virus, Varicella zoster virus, Cytomegalovirus, cat-scratch disease, Lyme disease, toxoplasmosis, toxocariasis, juvenile idiopathic arthritis, tubulointerstitial nephritis-uveitis, syphilis, and sarcoidosis, and each test was negative. He was initially treated with systemic azithromycin and valacyclovir but did not improve. As a result, he then underwent surgical exploration and vitrectomy with intravitreal injections of vancomycin, voriconazole, ceftazidime, and foscarnet. Although no signs of trauma or other injury were found during surgery, a hairlike intravitreal IOFB discovered during surgery was removed and sent to pathology. This IOFB was confirmed by pathology to be an eyelash. Vitreous cultures and PCR revealed methicillin sensitive Staphylococcus aureus. The patient was treated postoperatively with systemic dicloxacillin $500 \mathrm{mg}$ four times daily, prednisone $20 \mathrm{mg}$ daily with a rapid taper along with topical $1 \%$ atropine daily, and difluprednate $0.05 \%$ four times daily. Three weeks following surgery, the patient was pain-free, and his ocular inflammation was reduced to only $1+$ cell in the AC. He later developed a total tractional retinal detachment that required surgical repair. Over the following 2 years the patient remained pain-free, and the eye remained quiet with hand motions VA in his left eye.

\section{Case 3}

A 13 year old boy initially presented to an external provider with 3 weeks of redness, pain, photophobia, floaters in his left eye, and with a VA of 20/60. Despite topical and 


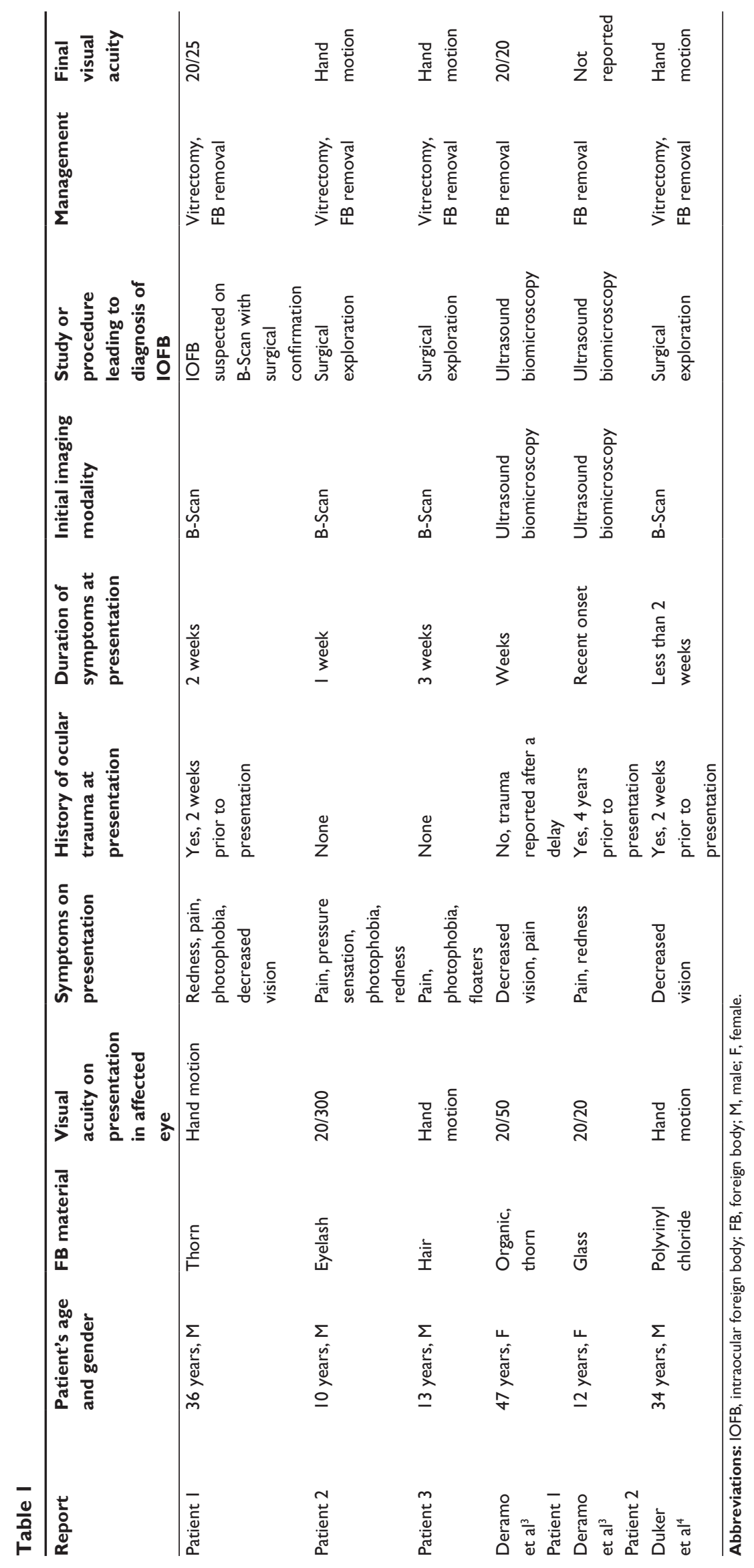


systemic corticosteroids, the patient's VA worsened. As a result, he was referred for further evaluation and management to a tertiary care center. Despite extensive questioning of both parents and patient, the patient denied any history of trauma, and the patient had no systemic symptoms. Initially he was treated with cycloplegics and topical steroids. Later, $40 \mathrm{mg} /$ day of oral prednisone and double strength Bactrim were added to cover possible toxoplasmosis due to a history of exposure to cats. Despite treatment, his vision and symptoms worsened. Examination revealed a normal OD. His left eye had hand motions VA with a nasal visual field deficit, pressure of 9, conjunctival injection, 4+ AC cell, $0.5 \mathrm{~mm}$ hypopyon, pupillary membranes, and no view to the posterior pole. B-scan ultrasound revealed a diffusely thickened choroid, vitreous debris, subretinal fluid, posterior vitreous detachment, and areas of minor traction but no apparent foreign body. Laboratory work up was negative for sarcoidosis, Lyme disease, HLA B27, toxoplasmosis, and Bartonella. Further treatment consisted of $80 \mathrm{mg}$ of oral prednisone daily, difluprednate $0.05 \%$ four times daily, and atropine $1 \%$ twice daily. The patient failed to improve, so he underwent pars plana vitrectomy, lensectomy, vitreous biopsy, intravitreal injection of antibiotics (vancomycin and amikacin), endolaser, and silicone oil injection. A foreign body (histologically consistent with hair) was discovered in the vitreous and removed. Gram stain and cultures were negative. His postoperative course was complicated by a total retinal detachment requiring a second surgery which was unsuccessful. On follow-up, the patient denied pain, his VA remained at the hand motions level, and there was no evidence of ongoing inflammation on exam.

\section{Discussion}

Intraocular foreign bodies carry a high risk of complications, especially when not detected promptly. In adults, there may be an eye injury that is not immediately brought to a physician's attention. In the authors' experience, children may deny trauma altogether to protect friends and relatives from getting into trouble. In our report, as well as in the majority of reviewed cases of nonmetallic IOFBs, patients present in the setting of intraocular inflammation (Table 1). This complicates IOFB visualization and identification, often leading to a missed diagnosis. B-scan ultrasonography is frequently ordered in this setting as a first line imaging study but has a suboptimal sensitivity for echo lucent foreign bodies and can contribute to an erroneous diagnostic impression. ${ }^{5-8}$ Orbital computed tomography (CT) scan offers relatively high sensitivity for
IOFBs, as has been reported by multiple investigators. ${ }^{5,-11}$ However, the majority of evidence in favor of $\mathrm{CT}$ as the imaging modality of choice for detection of IOFB comes from reports of metallic or otherwise radio-opaque (glass or stone) foreign bodies. In cases of less radiodense foreign bodies, CT imaging has reduced sensitivity., ${ }^{4,13}$ Moreover, CT may fail to detect even small ferromagnetic intraocular foreign bodies. ${ }^{14,15}$ Therefore, the occult IOFBs presented in this series may have been missed due to their radiolucency as has been reported with similar foreign bodies..$^{13}$

A clinical history with particular attention to traumatic events involving the eye holds the key to correct diagnosis and management. However, in some cases such history is difficult to elicit - two of our patients denied eye trauma, despite extensive questioning. In cases without a history of antecedent trauma, atypical features such as poor response to steroids should alert a physician to the possibility of IOFB. Therefore, a high index of suspicion for an occult IOFB should be maintained in cases of atypical uveitis with or without a history of eye injury.

\section{Disclosure}

The authors report no conflicts of interest in this work.

\section{References}

1. Jonas JB, Budde WM. Early versus late removal of retained intraocular foreign bodies. Retina. 1999;19(3):193-197.

2. Parke DW 3rd, Pathengay A, Flynn HW Jr, Albini T, Schwartz SG. Risk factors for endophthalmitis and retinal detachment with retained intraocular foreign bodies. J Ophthalmol. 2012;2012:758526.

3. Deramo VA, Shah GK, Baumal CR, et al. Ultrasound biomicroscopy as a tool for detecting and localizing occult foreign bodies after ocular trauma. Ophthalmology. 1999;106(2):301-305.

4. Duker JS, Fischer DH. Occult plastic intraocular foreign body. Ophthalmic Surg. 1989;20(3):169-170.

5. Patel SN, Langer PD, Zarbin MA, Bhagat N. Diagnostic value of clinical examination and radiographic imaging in identification of intraocular foreign bodies in open globe injury. Eur J Ophthalmol. 2012;22(2): 259-268.

6. Henrikson GC, Mafee MF, Flanders AE, Kriz RJ, Peyman GA. CT evaluation of plastic intraocular foreign bodies. AJNR Am J Neuroradiol. 1987;8(2):387-389.

7. Wilson WB, Preisbach JN, Lattin DE, Stears JC. Magnetic resonance imaging of nonmetallic orbital foreigh bodies. Am J Ophthalmol. 1988;105:612-617.

8. Das T, Namperumalsamy P. Ultrasonography in ocular trauma. Indian J Ophthalmol. 1987;85(3):121-125.

9. Papadopoulos A, Fotinos A, Maniatis V, et al. Assessment of intraocular foreign bodies by helical-CT multiplanar imaging. Eur Radiol. 2001; 11(8):1502-1510.

10. Dass AB, Ferrone PJ, Chu YR, Esposito M, Gray L. Sensitivity of spiral computed tomography scanning for detecting intraocular foreign bodies. Ophthalmology. 2001;108(12):2326-2328.

11. Lakits A, Prokesch R, Scholda C, Bankier A. Orbital helical computed tomography in the diagnosis and management of eye trauma. Ophthalmology. 1999;106(12):2330-2335. 
12. Topilow HW, Ackerman AL, Zimmerman RD. Limitations of computerized tomography in the localization of intraocular foreign bodies. Ophthalmology. 1984;91(9):1086-1091.

13. Seawright AA, Bourke RD, Gray PJ, Cooling RJ. Intravitreal cilia in phakic penetrating eye injury. Aust N Z J Ophthalmol. 1997;25(2): $133-135$.
14. Barnes E, Griffiths M, Elliott A. Intraocular foreign body missed by computed tomography. BMJ. 1993;306(6891):1542.

15. Deramo VA, Shah GK, Baumal CR, et al. The role of ultrasound biomicroscopy in ocular trauma. Trans Am Ophthalmol Soc. 1998;96: $355-365$.

\section{Publish your work in this journal}

Clinical Ophthalmology is an international, peer-reviewed journal covering all subspecialties within ophthalmology. Key topics include: Optometry; Visual science; Pharmacology and drug therapy in eye diseases; Basic Sciences; Primary and Secondary eye care; Patien Safety and Quality of Care Improvements. This journal is indexed on

Submit your manuscript here: http://www.dovepress.com/clinical-ophthalmology-journal

\section{Dovepress}

PubMed Central and CAS, and is the official journal of The Society of Clinical Ophthalmology (SCO). The manuscript management system is completely online and includes a very quick and fair peer-review system, which is all easy to use. Visit http://www.dovepress.com/ testimonials.php to read real quotes from published authors. 